Moroccan J. of Pure and Appl. Anal. (MJPAA)

Volume 7(1), 2021, Pages 50-65

ISSN: Online 2351-8227 - Print 2605-6364

DOI: $10.2478 / \mathrm{mjpaa}-2021-0006$

\title{
Existence result for a nonlinear elliptic problem by topological degree in Sobolev spaces with variable exponent
}

\author{
Mustapha AIT HAMMOU ${ }^{1}$, ElHoussine AZROUL ${ }^{2}$
}

Aвstract. The aim of this paper is to establish the existence of solutions for a nonlinear elliptic problem of the form

$$
\left\{\begin{array}{lll}
A(u)=f & \text { in } & \Omega \\
u=0 & \text { on } & \partial \Omega
\end{array}\right.
$$

where $A(u)=-\operatorname{div} a(x, u, \nabla u)$ is a Leray-Lions operator and $f \in W^{-1, p^{\prime}(.)}(\Omega)$ with $p(x) \in(1, \infty)$.

Our technical approach is based on topological degree method and the theory of variable exponent Sobolev spaces.

Mathematics Subject Classification (2020). 35J66, 47H11, 46E35.

Key words and phrases. Nonlinear elliptic problem, Sobolev spaces with variable exponent, Topological degree, Normalising maps.

Received : 26 April 2020 - Accepted: 30 October 2020.

(C) The Author(s) 2020. This article is published with open access by Sidi Mohamed Ben Abdallah University.

${ }^{1}$ Department of Mathematics, Laboratory LAMA, Faculty of Sciences Dhar El Mahraz, Sidi Mohamed Ben Abdellah University, Fez, Morocco.

e-mail: mustapha.aithammou@usmba.ac.ma (Corresponding Author).

${ }^{2}$ Department of Mathematics, Laboratory LAMA, Faculty of Sciences Dhar El Mahraz, Sidi Mohamed Ben Abdellah University, Fez, Morocco.

e-mail: elhoussine.azroul@usmba.ac.ma. 


\section{Introduction}

In this paper we are concerned with the following problem

$$
\left\{\begin{array}{lll}
A(u)=f & \text { in } & \Omega \\
u=0 & \text { on } & \partial \Omega
\end{array}\right.
$$

where $\Omega \subset \mathbb{R}^{N}(N \geq 1)$ is a bounded domain with a smooth boundary $\partial \Omega, A$ is a LerayLions operator in the form $A(u)=-\operatorname{div} a(x, u, \nabla u)$ defined on a subset of $W_{0}^{1, p(.)}(\Omega)$ and $f \in W^{-1, p^{\prime}(.)}(\Omega)$ where $p$ is a continuous function on $\bar{\Omega}$ such that

$$
1<p^{-}:=\inf _{x \in \bar{\Omega}} p(x) \leq p^{+}:=\sup _{x \in \bar{\Omega}} p(x)<\infty
$$

The goal of the paper is to prove the existence of solutions for the problem (1.1) in the variational framework by applying the topological degree constructed by Ait Hammou and Azroul in [1].

The use of topological degree theory is an effective tool in the study of nonlinear equations. As the most important stages in the history of this theory, Brouwer [11] had introduced it for continuous maps in finite dimensional Euclidean space. Leray and Schauder [20] developed it for compact operators in infinite dimensional Banach spaces. Browder [12, 13] introduced a degree theory for monotone type operators in reflexive Banach spaces. Berkovits [9] defined a topological degree for operators of generalized $\left(S_{+}\right)$type as an extension of the Leray-Schauder degree in order to solve abstract Hammerstein equation. This last degree is recently used for the study of some nonlinear elliptic problems in Sobolev spaces with variable exponent without and with weight $[2,3,4,5]$.

Many authors consider the existence of solutions for (1.1) by different methods in some spaces. Existence result of this kinds of problems have been given firstly by Boccardo-Galloüet [10] in the setting of classical Sobolev spaces $W^{1, p}(\Omega)$ where the growth of the function $a$ with respect to $u$ and $\nabla u$ was assumed to be polynominal. However, Dong [16] proved the existence of solution to problem (1.1) in the setting of Orlicz-Sobolev space $W^{1} L_{M}$ where $L_{M}$ is an Orlicz space and $M$, the $\mathrm{N}$-function that defines $L_{M}$, is related to the growth of the function $a$. The same results are also studied by Azroul et al [7] in the framework of Sobolev spaces with variable exponent using the theory of the calculus of variations and pseudo-monotonicity, in the unilateral case. In [6], the authors proved the existence of solutions for the problem (1.1) in the setting of Musielak-Orlicz-Sobolev space $W^{1} L_{\varphi}(\Omega)$, under the assumption that the conjugate function $\psi$ of $\varphi$ has the $\Delta_{2}$ property.

Our proposal to study this problem in the context of Sobolev spaces with variable exponent is motivated by his modeling of several physical phenomena, especially those related to nonNewtonian fluids [22].

This article is organized as follows: In section 2, we recall some basic results on the theory of Lebesgue-Sobolev spaces with variable exponent. The third section we introduce some classes of operators and topological degree. In section 4, we state and prove our main results. 


\section{Mathematical Preliminaries}

In the sequel, let $\Omega \subset \mathbb{R}^{N}(N \geq 1)$ be a bounded domain with a smooth boundary $\partial \Omega$.

We recall in this section some definitions and basic properties of the variable exponent Lebesgue and Sobolev spaces $L^{p(.)}(\Omega)$ and $W_{0}^{1, p(.)}(\Omega)$ (see $[8,15,17,18]$ for more details).

Denote

$$
C_{+}(\bar{\Omega})=\left\{h \in C(\bar{\Omega}) ; \inf _{x \in \bar{\Omega}} h(x)>1\right\} .
$$

For any $h \in C_{+}(\bar{\Omega})$, we define

$$
h^{-}:=\min \{h(x), x \in \bar{\Omega}\}, h^{+}:=\max \{h(x), x \in \bar{\Omega}\} .
$$

In this paper, we suppose that $p \in C_{+}(\bar{\Omega})$ such that

$$
1<p^{-} \leq p(x) \leq p^{+}<\infty
$$

and $p(\cdot)$ satisfies the log-Hölder continuity condition, i.e. there exists $\alpha>0$ such that for all $x, y \in \Omega, x \neq y$, one has

$$
|p(x)-p(y)| \leq \frac{\alpha}{\log (e+1 /|x-y|)}
$$

We define the variable exponent Lebesgue space

$$
L^{p(\cdot)}(\Omega)=\left\{u ; u: \Omega \rightarrow \mathbb{R} \text { is measurable and } \rho_{p(\cdot)}(u)<\infty\right\} .
$$

where

$$
\rho_{p(\cdot)}(u)=\int_{\Omega}|u(x)|^{p(x)} d x .
$$

We define a norm, the so-called Luxemburg norm, on this space by

$$
\|u\|_{p(\cdot)}=\inf \left\{\lambda>0 / \rho_{p(.)}\left(\frac{u}{\lambda}\right) \leq 1\right\} .
$$

Endowed with the Luxembourg norm, $L^{p(\cdot)}$ is a Banach spaces, separable, reflexive and uniformly convex.

The conjugate space of $L^{p(\cdot)}(\Omega)$ is $L^{p^{\prime}(\cdot)}(\Omega)$ where $\frac{1}{p(x)}+\frac{1}{p^{\prime}(x)}=1$. For any $u \in L^{p(\cdot)}(\Omega)$ and $v \in L^{p^{\prime}(\cdot)}(\Omega)$, Hölder inequality holds

$$
\left|\int_{\Omega} u v d x\right| \leq\left(\frac{1}{p^{-}}+\frac{1}{p^{\prime^{\prime}}}\right)\|u\|_{p(\cdot)}\|v\|_{p^{\prime}(\cdot)} \leq 2\|u\|_{p(\cdot)}\|v\|_{p^{\prime}(\cdot)} .
$$

If $p(\cdot), q(\cdot) \in C_{+}(\bar{\Omega}), q(\cdot) \leq p(\cdot)$ a.e. in $\Omega$ then there exists the continuous embedding $L^{p(\cdot)}(\Omega) \rightarrow L^{q(\cdot)}(\Omega)$.

We say that a sequence $\left(u_{n}\right) \subset L^{p(\cdot)}(\Omega)$ converges to $u \in L^{p(\cdot)}(\Omega)$ in the modular sense, denote $u_{n} \stackrel{\text { mod }}{\longrightarrow} u$ in $L^{p(\cdot)}$, if there exists $\lambda>0$ such that

$$
\rho_{p(\cdot)}\left(\frac{u_{n}-u}{\lambda}\right) \rightarrow 0 \text {, when } n \rightarrow \infty \text {. }
$$

Next, we define

$$
W^{1, p(\cdot)}(\Omega)=\left\{u \in L^{p(\cdot)}(\Omega) /|\nabla u| \in L^{p(\cdot)}(\Omega)\right\}
$$


with the norm

$$
\|u\|_{W^{1, p(\cdot)}}=\|u\|_{p(\cdot)}+\|\nabla u\|_{p(\cdot)}
$$

and $W_{0}^{1, p(\cdot)}(\Omega)$ as the closure of $C_{0}^{\infty}(\Omega)$ in $W^{1, p(\cdot)}(\Omega)$. By the Poincare inequality: there is a positive constant $C$, such that

$$
\|u\|_{p(\cdot)} \leq C\|\nabla u\|_{p(\cdot)}, \quad \forall u \in W_{0}^{1, p(\cdot)}(\Omega),
$$

$W_{0}^{1, p(\cdot)}(\Omega)$ has a norm

$$
\|u\|_{1, p(\cdot)}=\|\nabla u\|_{p(\cdot)}
$$

which is equivalent to the norm $\|\cdot\|_{W^{1, p(\cdot)}}$.

The space $\left(W_{0}^{1, p(\cdot)}(\Omega),\|\cdot\|_{1, p(\cdot)}\right)$ is a Banach space separable and reflexive .

We note that if $q \in C_{+}(\bar{\Omega})$ and $q(x)<p^{*}(x)$ for all $x \in \bar{\Omega}$ then the embedding $W_{0}^{1, p(\cdot)}(\Omega) \hookrightarrow L^{q(\cdot)}(\Omega)$ is compact and continuous, where $p^{*}(x)=\frac{N p(x)}{N-p(x)}$ if $p(x)<N$ and $p^{*}(x)=+\infty$ if $p(x) \geq N$. Moreover we have the compact embedding $W_{0}^{1, p(\cdot)}(\Omega) \hookrightarrow L^{p(\cdot)}(\Omega)$. We say that a sequence $\left(u_{n}\right) \subset W^{1, p(\cdot)}(\Omega)$ converges to $u \in W^{1, p(\cdot)}(\Omega)$ in the modular sense, denote $u_{n} \stackrel{\text { mod }}{\longrightarrow} u$ in $W^{1, p(\cdot)}$, if there exists $\lambda>0$ such that

$$
\rho_{p(\cdot)}\left(\frac{\nabla u_{n}-\nabla u}{\lambda}\right) \rightarrow 0, \text { when } n \rightarrow \infty .
$$

For any $v \in\left[W_{0}^{1, p(\cdot)}(\Omega)\right]^{\prime}$, there exists $\left(v_{0}, v\right) \in L^{p^{\prime}(\cdot)} \times\left[L^{p^{\prime}(\cdot)}\right]^{N}$ such that, for any $u \in W_{0}^{1, p(\cdot)}(\Omega)$,

$$
\langle v, u\rangle=\int_{\Omega}\left(v_{0} u+v \cdot \nabla u\right) d x .
$$

In other words, the dual space of $W_{0}^{1, p(\cdot)}(\Omega)$, denoted $W^{-1, p^{\prime}(\cdot)}(\Omega)$, is equipped with the norm

$$
\|v\|=\inf \left\{\left\|v_{0}\right\|_{p^{\prime}(\cdot)}+\sum_{i=1}^{N}\left\|v_{i}\right\|_{p^{\prime}(\cdot)}\right\},
$$

where the infinimum is taken on all possible decompositions $v=v_{0}-\operatorname{div} F$ with $v_{0} \in L^{p^{\prime}(\cdot)}(\Omega)$ and $F=\left(v_{1}, \ldots, v_{N}\right) \in\left(L^{p^{\prime}(\cdot)}(\Omega)\right)^{N}$.

We say that a sequence $\left(u_{n}\right) \subset W^{-1, p^{\prime}(\cdot)}(\Omega)$ converges to $u \in W^{-1, p^{\prime}(\cdot)}(\Omega)$ in the modular sense, denote $u_{n} \stackrel{\bmod }{\longrightarrow} u$ in $W^{-1, p^{\prime}(\cdot)}$, if $u_{n}$ and $u$ have representations

$$
u_{n}=g_{0}^{(n)}-\operatorname{divg}(n), u=g_{0}-\operatorname{divg}
$$

such that $g_{i}^{(n)}, g_{i} \in L^{p^{\prime}(\cdot)}(\Omega)$ and $g_{i}^{(n)} \stackrel{\text { mod }}{\longrightarrow} g_{i}$ in $L^{p^{\prime}(\cdot)}$ for $i=1,2, \ldots, N$.

If $\left(u_{n}\right) \subset L^{p(\cdot)}(\Omega)$ and $u \in L^{p(\cdot)}(\Omega)$, then the following relations hold true

$$
\begin{aligned}
& \|u\|_{p(\cdot)}>1 \quad \Rightarrow \quad\|u\|_{p(\cdot)}^{p^{-}} \leq \rho_{p(\cdot)}(u) \leq\|u\|_{p(\cdot)}^{p^{+}} \\
& \|u\|_{p(\cdot)}<1 \quad \Rightarrow \quad\|u\|_{p(\cdot)}^{p^{+}} \leq \rho_{p(\cdot)}(u) \leq\|u\|_{p(\cdot)}^{p^{-}}
\end{aligned}
$$




$$
\lim _{n \rightarrow \infty}\left\|u_{n}-u\right\|_{p(\cdot)}=0 \Leftrightarrow \lim _{n \rightarrow \infty} \rho_{p(\cdot)}\left(u_{n}-u\right)=0
$$

From (2.2), we can deduce that norm convergence and modular convergence are equivalent.

We will need to use the following two lemmas

Lemma 2.1. If $\left(u_{n}\right) \subset L^{p(\cdot)}(\Omega),\left(v_{n}\right) \subset L^{p^{\prime}(\cdot)}(\Omega), u_{n} \rightarrow u \in L^{p(\cdot)}(\Omega)$ in $L^{p(\cdot)}(\Omega)$ and $v_{n} \rightarrow v \in L^{p^{\prime}(\cdot)}(\Omega)$ a.e. and $v_{n} \rightarrow v$ in $L^{p^{\prime}(\cdot)}(\Omega)$, then $u_{n} v_{n} \rightarrow u v$ in $L^{1}(\Omega)$.

Lemma 2.2. If $\left(u_{n}\right) \subset L^{p(\cdot)}(\Omega)$ with $u_{n} \rightarrow u \in L^{p(\cdot)}(\Omega)$ a.e., $u_{n}, u \geq 0$ a.e. and $\left|c u_{n}(x)\right|^{p(x)} \leq h(x)$ a.e. for some $h \in L^{1}(\Omega)$ and $c>0$ then $u_{n} \rightarrow u$ in $L^{p(\cdot)}(\Omega)$.

\section{Some classes of operators and topological degree}

Definition 3.1. A mapping $F: D_{F} \subset W_{0}^{1, p(\cdot)}(\Omega) \rightarrow W^{-1, p^{\prime}(\cdot)}(\Omega)$ is

- pseudomonotone, denote $F \in(P M)$, if the conditions

$\left(u_{n}\right) \subset D_{F}, u_{n} \rightarrow u$ in $W_{0}^{1, p(\cdot)}(\Omega), F\left(u_{n}\right) \rightarrow \chi$ in $W^{-1, p^{\prime}(\cdot)}(\Omega)$ and

$\limsup _{n \rightarrow \infty}\left\langle F\left(u_{n}\right), u_{n}\right\rangle \leq\langle\chi, u\rangle$

imply that $u \in D_{F}, \chi=F(u)$ and $\left\langle F\left(u_{n}\right), u_{n}\right\rangle \rightarrow\langle F(u), u\rangle$.

- of the class $(M O D)$, denote $F \in(M O D)$, if the conditions

$\left(u_{n}\right) \subset D_{F}, u_{n} \rightarrow u$ in $W_{0}^{1, p(\cdot)}(\Omega), F\left(u_{n}\right) \rightarrow \chi$ in $W^{-1, p^{\prime}(\cdot)}(\Omega)$ and

$\limsup _{n \rightarrow \infty}\left\langle F\left(u_{n}\right), u_{n}\right\rangle \leq\langle\chi, u\rangle$

imply that $u \in D_{F}, \chi=F(u)$ and there exists a subsequence $\left(u_{n^{\prime}}\right)$ such that $u_{n^{\prime}} \stackrel{\text { mod }}{\longrightarrow} u$ in $W_{0}^{1, p(\cdot)}(\Omega)$ and $F\left(u_{n^{\prime}}\right) \stackrel{\text { mod }}{\longrightarrow} F(u)$ in $W^{-1, p^{\prime}(\cdot)}(\Omega)$.

- strongly quasibounded, denote $F \in(Q B)$, if the conditions

$\left(u_{n}\right) \subset D_{F}$ bounded and $\limsup _{n \rightarrow \infty}\left\langle F\left(u_{n}\right), u_{n}-\bar{u}\right\rangle$ is bounded from above for some $\bar{u} \in W_{0}^{1, p(\cdot)}(\Omega)$

imply that $\left(F\left(u_{n}\right)\right)$ is bounded in $W^{-1, p^{\prime}(\cdot)}(\Omega)$.

Let $Y=W_{0}^{1, p(\cdot)}(\Omega)$ and $Z=Y^{*}=W^{-1, p^{\prime}(\cdot)}(\Omega)$. We define the class $\mathcal{F}$ of admissible mappings and the class $\mathcal{H}$ of admissible homotopies as follows:

$F: D_{F} \subset Y \rightarrow Z$ belongs to $\mathcal{F}$, if

(a): $F$ is a strongly quasibounded mapping of the class $(M O D)$.

$F: D_{F} \subset Y \rightarrow Z$ belongs to $\mathcal{F}^{a}$, if $F \in \mathcal{F}$ and the following conditions hold:

(b): if $\left(u_{n}\right) \subset D_{F}$ is bounded, $t_{n} \rightarrow 0^{+}$and $\left\langle t_{n} F\left(u_{n}\right), u_{n}-\bar{u}\right\rangle$ is bounded from above for some $\bar{u} \in Y$, then $\left(t_{n} F\left(u_{n}\right)\right) \subset Z$ is bounded,

(c): if $\left(u_{n}\right) \subset D_{F}, u_{n} \rightarrow u \in Y, t_{n} \rightarrow 0^{+}, t_{n} F\left(u_{n}\right) \rightarrow \chi \in Z$ and $\limsup \left\langle t_{n} F\left(u_{n}\right), u_{n}\right\rangle \leq\langle\chi, u\rangle$, then $\left\langle t_{n} F\left(u_{n}\right), u_{n}\right\rangle \rightarrow\langle\chi, u\rangle$,

(d): if $\left(u_{n}\right) \subset D_{F}, u_{n} \rightarrow u$ in $Y, t_{n} \rightarrow 0^{+}, t_{n} F\left(u_{n}\right) \rightarrow \chi$ in $Z$ and $\limsup \left\langle t_{n} F\left(u_{n}\right), u_{n}\right\rangle \leq\langle\chi, u\rangle$, then $t_{n} F\left(u_{n}\right) \rightarrow 0$ in $Z$.

The homotopy $H: D_{H} \rightarrow Z$ belongs to $\mathcal{H}$, if $H$ is a strongly quasibounded homotopy of the class $(M O D)$. 
Lemma 3.1 ([1, Lemma 3.4]). If $F, G \in \mathcal{F}^{a}$, then $H(t, u)=t F(u)+(1-t) G(u)$ belongs to $\mathcal{H}$ with

$$
D_{H_{t}}=\left\{\begin{array}{cl}
D_{F} \cap D_{G} & \text { if } 0<t<1, \\
D_{G} & \text { if } t=0, \\
D_{F} & \text { if } t=1 .
\end{array}\right.
$$

Theorem 3.1 ([1, Remark 3.5]). For $F \in \mathcal{F}, G \subset Y$ open and bounded in $Y, f \in Z$ and $f \notin F\left(\partial_{Y} G\right)$ there exists an integer $d(F, G, f)$ (which is the degree function) satisfying the conditions:

(1) (Existence) if $d(F, G, f) \neq 0$, then $f \in F(G)$,

(2) (Additivity) if $G_{1}, G_{2} \in G$ are open and bounded, $f \notin F\left(\bar{G} \backslash\left(G_{1} \cup G_{2}\right)\right), G_{1} \cap G_{2}=\varnothing$, then

$$
d(F, G, f)=d\left(F, G_{1}, f\right)+d\left(F, G_{2}, f\right),
$$

(3) (Homotopy invariance) if $H \in \mathcal{H}, f \in Z$ and $f \notin H\left([0,1] \times \partial_{Y} G\right)$, then

$$
d(H(t, .), G, f)=\text { constant for all } t \in[0,1],
$$

(4) (Normalization) There exists a normalising map $K \in \mathcal{F}^{a}$ such that if $f \in Z, f \notin K\left(\partial_{Y} G\right)$ and $f \in K(G)$, then

$$
d(K, G, f)=1 \text {. }
$$

Any mapping $K \in \mathcal{F}^{a}$ satisfying

$$
\langle K(u), u\rangle>0 \text {, when } u \neq 0 \text {, and } K(0)=0
$$

can be chosen as a normalising map.

Using the conditions (1) - (4) for the degree function, we ca deduce, as in [1], some standard properties of the degree.

Property 1 ([1, Property 1]). Let $F, T \in \mathcal{F}^{a}, G \subset Y$ open and bounded in $Y, F / \partial_{Y} G=T / \partial_{Y} G$ and $f \in Z$. If $f \notin F\left(\partial_{Y} G\right)$, then

$$
d(F, G, f)=d(T, G, f) .
$$

Property 2 ([1, Property 3]). Let $F \in \mathcal{F}, G \subset Y$ open and bounded in $Y$ and $u_{0} \in G$. Define a mapping

$s: Y \rightarrow Y, s(u)=u-u_{0}$. If $0 \notin F\left(\partial_{Y} G\right)$, then

$$
d(F, G, 0)=d\left(F o s^{-1}, s(G), 0\right) .
$$

\section{Main result}

4.1. Basic assumptions and technical Lemmas. Let $A: W_{0}^{1, p(\cdot)}(\Omega) \rightarrow W^{-1, p^{\prime}(\cdot)}(\Omega)$ be the nonlinear operator of Leray-Lions, which is defined by

$$
A(u)=-\operatorname{div} a(x, u, \nabla u) .
$$

i.e.

$$
\langle A(u), v\rangle=\int_{\Omega} a(x, u, \nabla u) . \nabla v d x \text { for all } v \in W_{0}^{1, p(.)}(\Omega) .
$$

The function $a$ is assumed to satisfy the conditions:

$\left(A_{1}\right): a: \Omega \times \mathbb{R} \times \mathbb{R}^{N} \rightarrow \mathbb{R}^{N}$ is a Caratheodory function,i.e., $a(., \eta, \xi)$ is measurable on $\Omega$ for all $(\eta, \xi) \in \mathbb{R} \times \mathbb{R}^{N}$ and $a(x, . .$.$) is continuous on \mathbb{R} \times \mathbb{R}^{N}$ for a.e. $x \in \Omega$. 
$\left(A_{2}\right)$ : There exist a positive function $b(x)$ in $L^{p^{\prime}(\cdot)}(\Omega)$ and constant $c>0$ such that $|a(x, \eta, \xi)| \leq b(x)+c\left(|\eta|^{p(x)-1}+|\xi|^{p(x)-1}\right)$ for all $(\eta, \xi) \in \mathbb{R} \times \mathbb{R}^{N}$ and a.e. $x \in \Omega$. $\left(A_{3}\right):\left(a(x, \eta, \xi)-a\left(x, \eta, \xi^{\prime}\right)\right) \cdot\left(\xi-\xi^{\prime}\right)>0$ a.e. $x \in \Omega$, for all $\eta \in \mathbb{R}$ and $\xi, \xi^{\prime} \in \mathbb{R}^{N}, \xi \neq \xi^{\prime}$. $\left(A_{4}\right)$ : There exist a constant $c^{\prime}>0$ such that $a(x, \eta, \xi) \cdot \xi \geq c^{\prime}|\xi|^{p(x)}$.

Lemma 4.1. Assume that $\left(A_{1}\right)-\left(A_{4}\right)$ hold, and let $\left(u_{n}\right)$ be a sequence in $W_{0}^{1, p(\cdot)}(\Omega)$ such that $u_{n} \rightarrow u$ in $W_{0}^{1, p(\cdot)}(\Omega)$ and $u_{n} \rightarrow$ u a.e in $\Omega$ and

$$
\int_{\Omega}\left[a\left(x, u_{n}, \nabla u_{n}\right)-a\left(x, u_{n}, \nabla u\right)\right] \cdot \nabla\left(u_{n}-u\right) d x \rightarrow 0 .
$$

Then $u_{n} \rightarrow$ u strongly in $W_{0}^{1, p(\cdot)}(\Omega)$.

Proof. See the proof of Lemma 3.2 in [19] taking the weight function $\rho \equiv 1$.

Lemma 4.2. Assume that the conditions $\left(A_{1}\right)-\left(A_{3}\right)$ hold. Then the operator A defined by (4.1) is pseudomonotone.

Proof. Let $\left(u_{n}\right)$ be a sequence in $W_{0}^{1, p(\cdot)}(\Omega)$ such that

$$
\left\{\begin{array}{l}
u_{n} \rightarrow u \text { in } W_{0}^{1, p(\cdot)}(\Omega) \\
A u_{n} \rightarrow \chi \text { in } W^{-1, p^{\prime}(\cdot)}(\Omega) \\
\limsup _{n \rightarrow \infty}\left\langle A u_{n}, u_{n}\right\rangle \leq\langle\chi, u\rangle .
\end{array}\right.
$$

We will prove that

$$
\chi=A u \text { and }\left\langle A u_{n}, u_{n}\right\rangle \longrightarrow\langle\chi, u\rangle \text { as } n \rightarrow+\infty .
$$

Since $\left(u_{n}\right)$ is a bounded sequence in $W_{0}^{1, p(\cdot)}(\Omega)$, then, by the reflexivity of $W_{0}^{1, p(\cdot)}(\Omega)$ and the compact embedding $W_{0}^{1, p(\cdot)}(\Omega) \hookrightarrow L^{p(\cdot)}(\Omega)$, there is a subsequence still denoted by $\left(u_{n}\right)$ such that

$$
\begin{gathered}
u_{n} \rightarrow u \text { in } W_{0}^{1, p(\cdot)}(\Omega), \\
u_{n} \rightarrow u \text { in } L^{p(\cdot)}(\Omega) \text { and a.e in } \Omega .
\end{gathered}
$$

By the growth condition $\left(A_{2}\right)$, the sequence $\left(a\left(x, u_{n}, \nabla u_{n}\right)\right)$ is bounded in $\left(L^{p^{\prime}(\cdot)}(\Omega)\right)^{N}$, therefore there exists a function $\varphi \in\left(L^{p^{\prime}(\cdot)}(\Omega)\right)^{N}$ such that

$$
a\left(x, u_{n}, \nabla u_{n}\right) \rightarrow \varphi \text { in } \quad\left(L^{p^{\prime}(\cdot)}(\Omega)\right)^{N} \text { as } n \rightarrow \infty .
$$

It is clear that, for all $v \in W_{0}^{1, p(\cdot)}(\Omega)$,

$$
\begin{aligned}
\langle\chi, v\rangle & =\lim _{n \rightarrow \infty}\left\langle A u_{n}, v\right\rangle \\
& =\lim _{n \rightarrow \infty} \int_{\Omega} a\left(x, u_{n}, \nabla u_{n}\right) \cdot \nabla v d x \\
& =\int_{\Omega} \varphi \cdot \nabla v d x .
\end{aligned}
$$


by combining (4.3) and (4.5), we have

$$
\limsup _{n \rightarrow \infty}\left\langle A\left(u_{n}\right), u_{n}\right\rangle=\limsup _{n \rightarrow \infty} \int_{\Omega} a\left(x, u_{n}, \nabla u_{n}\right) . \nabla u_{n} d x \leq \int_{\Omega} \varphi \cdot \nabla u d x .
$$

Therefore

$$
\limsup _{n \rightarrow \infty} \int_{\Omega} a\left(x, u_{n}, \nabla u_{n}\right) \cdot \nabla u_{n} d x \leq \int_{\Omega} \varphi \cdot \nabla u d x .
$$

On the other hand, thanks to assumption $\left(A_{3}\right)$ we have

$$
\int_{\Omega}\left(a\left(x, u_{n}, \nabla u_{n}\right)-a\left(x, u_{n}, \nabla u\right)\right) \cdot\left(\nabla u_{n}-\nabla u\right) d x>0 .
$$

then

$$
\begin{aligned}
\int_{\Omega} a\left(x, u_{n}, \nabla u_{n}\right) . \nabla u_{n} d x \geq & -\int_{\Omega} a\left(x, u_{n}, \nabla u\right) . \nabla u d x+\int_{\Omega} a\left(x, u_{n}, \nabla u_{n}\right) . \nabla u d x \\
& +\int_{\Omega} a\left(x, u_{n}, \nabla u\right) . \nabla u_{n} d x,
\end{aligned}
$$

by (4.4), we get

$$
\liminf _{n \rightarrow \infty} \int_{\Omega} a\left(x, u_{n}, \nabla u_{n}\right) . \nabla u_{n} d x \geq \int_{\Omega} \varphi \cdot \nabla u d x .
$$

This implies, by using (4.6) that

$$
\lim _{n \rightarrow \infty} \int_{\Omega} a\left(x, u_{n}, \nabla u_{n}\right) . \nabla u_{n} d x=\int_{\Omega} \varphi \cdot \nabla u d x .
$$

By combining (4.5) and (4.8), we obtain

$$
\left\langle A u_{n}, u_{n}\right\rangle \longrightarrow\langle\chi, u\rangle \text { as } n \rightarrow+\infty \text {. }
$$

On the other hand, by (4.8)), and the fact that $a\left(x, u_{n}, \nabla u\right) \longrightarrow a(x, u, \nabla u)$ in $\left(L^{p^{\prime}(\cdot)}(\Omega)\right)^{N}$ we deduce that

$$
\lim _{n \rightarrow+\infty} \int_{\Omega}\left(a\left(x, u_{n}, \nabla u_{n}\right)-a\left(x, u_{n}, \nabla u\right)\right) \cdot\left(\nabla u_{n}-\nabla u\right) d x=0
$$

by Lemma 4.1 , we obtain

$$
u_{n} \longrightarrow u \quad \text { in } W_{0}^{1, p(\cdot)}(\Omega),
$$

then, for a subsequence still denoted $\left(u_{n}\right)$ we have $\nabla u_{n} \rightarrow \nabla u$ a.e. in $\Omega$.

Since $a$ is Carathéodory function we can write

$$
a\left(x, u_{n}, \nabla u_{n}\right) \rightarrow a(x, u, \nabla u) \quad \text { in } \quad\left(L^{p^{\prime}(\cdot)}(\Omega)\right)^{N},
$$

by consequent, $\chi=A u$ which allows to conclude that the operator $A$ is pseudomonotone.

Lemma 4.3. Assume that the conditions $\left(A_{1}\right)-\left(A_{3}\right)$ hold. If the sequence $\left(u_{n}\right) \subset W_{0}^{1, p(\cdot)}(\Omega)$ is bounded, $\left(t_{n}\right) \subset[0,1]$ and $\left(\left\langle t_{n} A\left(u_{n}\right), u_{n}-\bar{u}\right\rangle\right)$ is bounded from above some $\bar{u} \in W_{0}^{1, p(\cdot)}(\Omega)$, then the sequence $\left(t_{n} a\left(x, u_{n}, \nabla u_{n}\right)\right)$ is bounded in $\left(L^{p^{\prime}(.)}(\Omega)\right)^{N}$. 
Proof. Let $w \in\left(L^{p(\cdot)}(\Omega)\right)^{N}$ be arbitrary. By condition $\left(A_{3}\right)$ we have:

$$
t_{n}\left(a\left(x, u_{n}, \nabla u_{n}\right)-a\left(x, u_{n}, w\right)\right) \cdot\left(\nabla u_{n}-w\right) \geq 0 .
$$

Consequently:

$$
\int_{\Omega} t_{n} a\left(x, u_{n}, \nabla u_{n}\right) \cdot(w-\nabla \bar{u}) d x \leq\left\langle t_{n} A\left(u_{n}\right), u_{n}-\bar{u}\right\rangle+\int_{\Omega} t_{n} a\left(x, u_{n}, w\right) \cdot\left(w-\nabla u_{n}\right) d x
$$

The first term on the right remains bounded from above by the assumption. Moreover, by $\left(A_{2}\right)$, we have:

$$
\left|a\left(x, u_{n}, w\right)\right|^{p^{\prime}(x)} \leq \operatorname{const}\left(|b(x)|^{p^{\prime}(x)}+\left|u_{n}\right|^{p(x)}+|w|^{p(x)}\right) .
$$

If $\left\|a\left(x, u_{n}, w\right)\right\|_{p^{\prime}(\cdot)} \leq 1$, then $\left\|t_{n} a\left(x, u_{n}, w\right)\right\|_{p(\cdot)} \leq 1$.

Suppose that $\left\|a\left(x, u_{n}, w\right)\right\|_{p^{\prime}(\cdot)}>1$, then we have by (2.1) and (4.10)

$$
\begin{aligned}
\left\|a\left(x, u_{n}, w\right)\right\|_{p^{\prime}(\cdot)}^{p^{\prime-}} & \leq \rho_{p^{\prime}(\cdot)}\left(a\left(x, u_{n}, w\right)\right) \\
& \leq \operatorname{const}\left(\rho_{p^{\prime}(\cdot)}(b)+\rho_{p(\cdot)}\left(u_{n}\right)+\rho_{p(\cdot)}(w)\right) .
\end{aligned}
$$

Hence $\left\{a\left(x, u_{n}, w\right)\right\}$ is bounded in $\left(L^{p^{\prime}(.)}(\Omega)\right)^{N}$, which implies that the second term on the right in (4.9) is also bounded. By the theorem of Banach-Steinhaus, the sequence $\left(t_{n} a\left(x, u_{n}, \nabla u_{n}\right)\right)$ remains bounded in $\left(L^{p^{\prime}(\cdot)}(\Omega)\right)^{N}$.

Lemma 4.4. Assume that the conditions $\left(A_{1}\right)-\left(A_{2}\right)$ hold. If the sequence $\left(u_{n}\right) \subset W_{0}^{1, p(\cdot)}(\Omega)$, $u_{n} \rightarrow u \in W_{0}^{1, p(\cdot)}$ in $W_{0}^{1, p(\cdot)}$, then $t_{n} a\left(x, u_{n}, \nabla u_{n}\right) \rightarrow \operatorname{ta}(x, u, \nabla u)$ in $\left(L^{p^{\prime}(\cdot)}(\Omega)\right)^{N}$.

Proof. Since $u_{n} \rightarrow u$ in $W_{0}^{1, p(\cdot)}(\Omega)$, then $u_{n} \rightarrow u$ and $\nabla u_{n} \rightarrow \nabla u$ in $L^{p(\cdot)}(\Omega)$. Hence there exist a subsequence $\left(u_{k}\right)$ of $\left(u_{n}\right)$ and measurable functions $h$ in $L^{p(\cdot)}(\Omega)$ and $g$ in $\left(L^{p(\cdot)}(\Omega)\right)^{N}$ such that

$$
\begin{gathered}
u_{k}(x) \rightarrow u(x) \text { and } \nabla u_{k}(x) \rightarrow \nabla u(x), \\
\left|u_{k}(x)\right| \leq h(x) \text { and }\left|\nabla u_{k}(x)\right| \leq g(x)
\end{gathered}
$$

for a.e. $x \in \Omega$ and all $k \in \mathbb{N}$. Since $a$ satisfies the Carathéodory condition and $t_{k} \rightarrow t$, we obtain that

$$
t_{k} a\left(x, u_{k}(x), \nabla u_{k}(x)\right) \rightarrow \operatorname{ta}(x, u(x), \nabla u(x)) \text { a.e. } x \in \Omega .
$$

It follows from $\left(A_{2}\right)$ that

$$
\left|t_{k} a\left(x, u_{k}(x), \nabla u_{k}(x)\right)\right| \leq b(x)+c\left(|h(x)|^{p(x)-1}+|g(x)|^{p(x)-1}\right)
$$

for a.e. $x \in \Omega$ and for all $k \in \mathbb{N}$. Since

$$
b+|h|^{p(x)-1}+|g(x)|^{p(x)-1} \in L^{p^{\prime}(x)}(\Omega),
$$

and taking into account the equality $\rho_{p^{\prime}(\cdot)}\left(t_{k} a\left(x, u_{k}(x), \nabla u_{k}(x)\right)-t a(x, u(x), \nabla u(x))\right)=\int_{\Omega}\left|t_{n} a\left(x, u_{k}(x), \nabla u_{k}(x)\right)-t a(x, u(x), \nabla u(x))\right|^{p^{\prime}(x)} d x$, the dominated convergence theorem and the equivalence (2.2) imply that

$$
t_{k} a\left(x, u_{k}, \nabla u_{k}\right) \rightarrow t a(x, u, \nabla u) \text { in }\left(L^{p^{\prime}(\cdot)}(\Omega)\right)^{N}
$$


By contradiction argument,

$$
t_{n} a\left(x, u_{n}, \nabla u_{n}\right) \rightarrow t a(x, u, \nabla u) \text { in }\left(L^{p^{\prime}(\cdot)}(\Omega)\right)^{N} .
$$

Lemma 4.5. Assume that the conditions $\left(A_{1}\right)-\left(A_{2}\right)$ hold. If the sequence $\left(u_{n}\right) \subset W_{0}^{1, p(\cdot)}(\Omega)$, $u_{n} \rightarrow u \in L^{p(\cdot)}$ a.e., $\nabla u_{n} \rightarrow \nabla u \in L^{p(\cdot)}$ a.e., $\nabla u_{n} \rightarrow \nabla u$ in $L^{p(\cdot)}(\Omega), t_{n} \rightarrow t \in[0,1]$ and $t_{n} a\left(x, u_{n}, \nabla u_{n}\right) \rightarrow t a(x, u, \nabla u)$ in $\left(L^{p^{\prime}(\cdot)}(\Omega)\right)^{N}$ then

$$
t_{n} a\left(x, u_{n}, \nabla u_{n}\right) . \nabla u_{n} \rightarrow t a(x, u, \nabla u) . \nabla u
$$

in $L^{1}(\Omega)$.

Proof. Denote $v_{n}(x)=t_{n} a\left(x, u_{n}, \nabla u_{n}\right)$ and $v=t a(x, u, \nabla u)$.

Since $u_{n} \rightarrow u$ a.e., $\nabla u_{n} \rightarrow \nabla u$ a.e. and $t_{n} \rightarrow t$ and in virtu to the assumption $\left(A_{2}\right)$, we deduce that $v_{n} \rightarrow v$ a.e.

We have also $v_{n} \rightarrow v$ in $\left(L^{p^{\prime}(\cdot)}(\Omega)\right)^{N}$ and $\nabla u_{n} \rightarrow \nabla u$ in $L^{p(\cdot)}(\Omega)$.

Then, by Lemma 2.1, $v_{n} . \nabla u_{n} \rightarrow v . \nabla u$ in $L^{1}(\Omega)$ i.e.

$$
t_{n} a\left(x, u_{n}, \nabla u_{n}\right) . \nabla u_{n} \rightarrow t a(x, u, \nabla u) . \nabla u
$$

in $L^{1}(\Omega)$.

Lemma 4.6. Assume that the conditions $\left(A_{1}\right)-\left(A_{3}\right)$ hold. If the sequence $\left(u_{n}\right) \subset W_{0}^{1, p(\cdot)}(\Omega)$, $u_{n} \rightarrow u$ in $W_{0}^{1, p(\cdot)}(\Omega),\left\{t_{n}\right\} \subset[0,1], t_{n} \rightarrow t, t_{n} A\left(u_{n}\right) \rightarrow \chi$ in $W^{-1, p^{\prime}(\cdot)}(\Omega)$ and limsup $\left\langle t_{n} A\left(u_{n}\right), u_{n}\right\rangle \leq\langle\chi, u\rangle$, then

$$
\left\langle t_{n} A\left(u_{n}\right), u_{n}\right\rangle \rightarrow\langle\chi, u\rangle .
$$

Proof. By a demonstration similar to that of Lemma 4.2, we can show that there exists a function $\varphi \in\left(L^{p^{\prime}(\cdot)}(\Omega)\right)^{N}$ such that

$$
\limsup _{n \rightarrow \infty} \int_{\Omega} t_{n} a\left(x, u_{n}, \nabla u_{n}\right) . \nabla u_{n} d x \leq \int_{\Omega} \varphi \cdot \nabla u d x .
$$

So that it is enough to prove that

$$
\liminf \int_{\Omega} t_{n} a\left(x, u_{n}, \nabla u_{n}\right) . \nabla u_{n} \geq \int_{\Omega} \varphi \cdot \nabla u .
$$

Denote

$$
\Omega_{k}=\{x \in \Omega /|\nabla u(x)| \leq k\}
$$

and

$$
E_{k}(x)= \begin{cases}1 & , \text { when } x \in \Omega_{k} \\ 0 & , \text { otherwise }\end{cases}
$$

By condition $\left(A_{3}\right)$ we have

$$
\int_{\Omega} t_{n}\left[a\left(x, u_{n}, E_{k}(x) \nabla u\right)-a\left(x, u_{n}, \nabla u_{n}\right)\right] .\left[E_{k}(x) \nabla u-\nabla u_{n}\right] \geq 0 .
$$


Consequently,

$$
\begin{aligned}
\int_{\Omega} t_{n} a\left(x, u_{n}, \nabla u_{n}\right) . \nabla u_{n} & \geq-\int_{\Omega} t_{n} a\left(x, u_{n}, E_{k}(x) \nabla_{u}\right) \cdot E_{k}(x) \nabla u \\
& +\int_{\Omega} t_{n} a\left(x, u_{n}, \nabla u_{n}\right) \cdot E_{k}(x) \nabla u+\int_{\Omega} t_{n} a\left(x, u_{n}, E_{k}(x) \nabla u\right) . \nabla u_{n} .
\end{aligned}
$$

From (4.11)-(4.13), we can deduce that the first term on the right in (4.14) tend towards

$$
-\int_{\Omega} t a\left(x, u, E_{k}(x) \nabla u\right) \cdot E_{k}(x) \nabla u,
$$

and the third term on the right in (4.14) will tend towards

$$
\int_{\Omega} t a\left(x, u, E_{k}(x) \nabla u\right) . \nabla u \text {. }
$$

Consequently,

$$
\begin{aligned}
\liminf \int_{\Omega} t_{n} a\left(x, u_{n}, \nabla u_{n}\right) \cdot \nabla u_{n} & \geq \liminf \int_{\Omega} t_{n} a\left(x, u_{n}, \nabla u_{n}\right) \cdot E_{k}(x) \nabla u+\int_{\Omega \backslash \Omega_{k}} t a(x, u, 0) . \nabla u \\
& =\int_{\Omega_{k}} x \cdot \nabla u+\int_{\Omega \backslash \Omega_{k}} t a(x, u, 0) . \nabla u,
\end{aligned}
$$

Letting $k \rightarrow \infty$, then $\Omega_{k} \rightarrow \Omega$, and hence

$$
\liminf \int_{\Omega} t_{n} a\left(x, u_{n}, \nabla u_{n}\right) . \nabla u_{n} \geq \int_{\Omega} x \cdot \nabla u
$$

Lemma 4.7. Assume that the conditions $\left(A_{1}\right)-\left(A_{4}\right)$ hold. If the sequence $\left(u_{n}\right) \subset W_{0}^{1, p(\cdot)}(\Omega)$, $u_{n} \rightarrow u \in W_{0}^{1, p(\cdot)}(\Omega)$ in $W_{0}^{1, p(\cdot)}(\Omega), A\left(u_{n}\right) \rightarrow \chi$ in $W^{-1, p^{\prime}(\cdot)}(\Omega)$ and $\limsup \left\langle A\left(u_{n}\right), u_{n}\right\rangle \leq\langle\chi, u\rangle$, then $u_{n} \rightarrow u$ in $W_{0}^{1, p(\cdot)}(\Omega)$ for some subsequence.

Proof. We deduce as in [21] that $\nabla u_{n}(x) \rightarrow \nabla u(x)$ a.e. for some subsequence. According to Lemma 4.3 we may assume that

$$
a\left(x, u_{n}, \nabla u_{n}\right) \rightarrow w(x) \text { in } L^{p^{\prime}(\cdot)} .
$$

Since

$$
a\left(x, u_{n}, \nabla u_{n}\right) \rightarrow a(x, u, \nabla u) \text { a.e., }
$$

then $w(x)=a(x, u, \nabla u)$ a.e. $A$ is pseudomonotone.

Hence

By Lemma 4.5,

$$
u \in W_{0}^{1, p(\cdot)}(\Omega), \chi=A(u) \text { and }\left\langle A\left(u_{n}\right), u_{n}\right\rangle \rightarrow\langle A(u), u\rangle .
$$

$$
a\left(x, u_{n}, \nabla u_{n}\right) . \nabla u_{n} \rightarrow a(x, u, \nabla u) . \nabla u
$$

in $L^{1}(\Omega)$, and by condition $\left(A_{4}\right)$,

$$
c^{\prime}\left|\nabla u_{n}(x)\right|^{p(x)} \leq\left(a\left(x, u_{n}(x), \nabla u_{n}(x)\right) . \nabla u_{n}(x) .\right.
$$

The right hand side converges in $L^{1}(\Omega)$ in accordance with Lemma 2.1. Lemma 2.2 implies $\nabla u_{n} \rightarrow \nabla u$ in $L^{p(\cdot)}(\Omega)$, and hence $u_{n} \rightarrow u$ in $W_{0}^{1, p(\cdot)}$. 
Theorem 4.1. If the conditions $\left(A_{1}\right)-\left(A_{4}\right)$ hold, then the mapping $A$ defined by (4.1) belong to the class $\mathcal{F}^{a}$.

Proof. Strong quasiboundedness and condition b) of class $\mathcal{F}^{a}$ follow immediately from Lemma 4.3. Lemma 4.6 implies condition c), and condition d) follows from Lemmas 4.6, 4.5 and 4.4. Suppose that

$$
\left(u_{n}\right) \subset W_{0}^{1, p(\cdot)}(\Omega), u_{n} \rightarrow u \text { in } W_{0}^{1, p(\cdot)}(\Omega), A\left(u_{n}\right) \rightarrow \chi \text { in } W^{-1, p^{\prime}(\cdot)}(\Omega) \text { and } \limsup _{n \rightarrow \infty}\left\langle A\left(u_{n}\right), u_{n}\right\rangle \leq\langle\chi, u\rangle .
$$

By pseudomonotonicity, $u \in W_{0}^{1, p(\cdot)}(\Omega), \chi=A(u)$ and $\left\langle A\left(u_{n}\right), u_{n}\right\rangle \rightarrow\langle A(u), u\rangle$. If $\left(A_{4}\right)$ holds, then, by Lemma $4.7, u_{n} \rightarrow u$ in $W_{0}^{1, p(\cdot)}(\Omega)$ for some subsequence. Choosing $t_{n}=1$ in Lemma 4.5 and Lemma 4.4 we may deduce that $A\left(u_{n}\right) \stackrel{\text { mod }}{\longrightarrow} A(u)$ in $W^{-1, p^{\prime}(\cdot)}(\Omega)$. Hence $A \in(M O D)$.

4.2. Normalising maps. Let $Y=W_{0}^{1, p(.)}(\Omega), Z=W^{-1, p^{\prime}(.)}(\Omega)$. We start with an abstract existence theorem.

Theorem 4.2. Let $G \subset Y$ be open and bounded in $Y, 0 \in G, f \in Z$ and $F \in \mathcal{F}^{a}$. Suppose that there exists a normalising map $K \in \mathcal{F}^{a}$ such that $K(0)=0$ and $\langle K(u), u\rangle \geq b>0$ for all $u \in \partial_{Y} G$. Choose a constant $a \geq 0$ such that

$$
a \leq \inf _{u \in \partial_{Y} G} \frac{\langle K(u), u\rangle}{\|K(u)\|} .
$$

If $\langle F(u)-f, u\rangle+\|F(u)-f\| a>0$ for all $u \in \partial_{Y} G$, then $d(F, G, f)=1$.

Proof. We may assume that $f=0$. Since $\langle K(u), u\rangle \geq b>0$ for all $u \in \partial_{Y} G$, it is clear that $0 \notin \overline{K\left(\partial_{Y} G\right)}$. Define by

$$
H(t, u)=t F(u)+(1-t) K(u) .
$$

a homotopy which belong to the class $\mathcal{H}$ by Lemma 3.1. We show that $H(t, u) \neq 0$ for all $t \in[0,1], u \in \partial_{Y} G$. If $0 \in H\left([0,1] \times \partial_{Y} G\right)$, then

$$
t F(u)+(1-t) K(u)=0
$$

for some $u \in \partial_{Y} G$ and $t \in[0,1]$. It is clear that $t \neq 0$. Thus

$$
t\|F(u)\|=(1-t)\|K(u)\|,
$$

implying

$$
1-\frac{1}{t}=-\frac{\|F(u)\|}{\|K(u)\|}
$$

On the other hand,

$$
t\langle F(u), u\rangle+(1-t)\langle K(u), u\rangle=0,
$$

and therefore

$$
\langle F(u), u\rangle=\left(1-\frac{1}{t}\right)\langle K(u), u\rangle=-\|F(u)\| \frac{\langle K(u), u\rangle}{\|K(u)\|} \leq-\|F(u)\| a,
$$


which is a contradiction. Hence $H(t, u) \neq 0$ for all $t \in[0,1]$ and $u \in \partial_{Y} G$. By homotopy invariance,

$$
d(F, G, 0)=f(K, G, 0) .
$$

Since $0 \in K(G)$, we have $d(K, G, 0)=1$.

Corollary 4.1. Let $G \subset Y$ be open and bounded in $Y, \bar{u} \in G, f \in Z$ and $F \in \mathcal{F}^{a}$. Suppose that there exists a normalising map $K \in \mathcal{F}^{a}$ such that $\tilde{K}(u)=K(u+\bar{u})-K(\bar{u})$ is also a normalising map in the class $\mathcal{F}^{a}$ and

$$
\langle K(u)-K(\bar{u}), u-\bar{u}\rangle \geq b>0 \text { for all } u \in \partial_{Y} G .
$$

Choose a constant $a \geq 0$ such that

$$
a \leq \inf _{u \in \partial_{Y} G} \frac{\langle K(u)-K(\bar{u}), u-\bar{u}\rangle}{\|K(u)-K(\bar{u})\|} .
$$

If $\langle F(u)-f, u-\bar{u}\rangle+\|F(u)-f\| a>0$ for all $u \in \partial_{Y} G$, then $d(F, G, f)=1$.

Proof. We may assume that $f=0$. It follows from the above assumptions that the degree $d(F, G, 0)$ is defined. Define $s(u)=u-\bar{u}$. Then, by Property 2,

$$
d(F, G, 0)=d\left(F o s^{-1}, s(G), 0\right) .
$$

Let $u \in \partial_{Y} s(G)$ be arbitrary. Denote $u=u^{\prime}-\bar{u}$, where $u^{\prime} \in \partial_{Y} G$. Now $\langle\tilde{K}(u), u\rangle=\left\langle K\left(u^{\prime}\right)-K(\bar{u}), u^{\prime}-\bar{u}\right\rangle \geq b>0$ and

$$
\frac{\langle\tilde{K}(u), u\rangle}{\|\tilde{K}(u)\|}=\frac{\left\langle K\left(u^{\prime}\right)-K(\bar{u}), u-\bar{u}\right\rangle}{\left\|K\left(u^{\prime}\right)-K(\bar{u})\right\|} \geq a \geq 0 .
$$

Moreover,

$$
\left\langle\operatorname{Fos}^{-1}(u), u\right\rangle+\left\|F o s^{-1}(u)\right\| a=\left\langle F\left(u^{\prime}\right), u^{\prime}-\bar{u}\right\rangle+\left\|F\left(u^{\prime}\right)\right\| a .
$$

Therefore the assumptions of Theorem 4.2 hold. Hence

$$
d\left(\operatorname{Fos}^{-1}(u), s(G), 0\right)=1 \text {. }
$$

Remark 4.1. The mapping, so-called $p(\cdot)$-Laplacian, $K: W_{0}^{1, p(\cdot)}(\Omega) \rightarrow W^{-1, p^{\prime}(\cdot)}(\Omega)$ defined by

$$
K(u)=-\Delta_{p(.)}(u)=-\operatorname{div}\left(|\nabla u(x)|^{p(x)-2} \nabla u(x)\right) .
$$

i.e.

$$
\langle K u, v\rangle=\int_{\Omega}|\nabla u(x)|^{p(x)-2} \nabla u(x) . \nabla v(x) d x \text { for all } v \in W_{0}^{1, p(\cdot)}(\Omega),
$$

belongs to the class $\mathcal{F}^{a},\langle K(u), u\rangle>0$ for $u \in W_{0}^{1, p(\cdot)}(\Omega), u \neq 0$ and $K(0)=0$.

Proof. Denote $a(x, \eta, \xi)=|\xi|^{p(x)-2} \xi$, then

$$
\langle K u, v\rangle=\int_{\Omega} a(x, u, \nabla u) . \nabla v d x \text { for all } v \in W_{0}^{1, p(\cdot)}(\Omega) .
$$


It is obvious that the conditions $\left(A_{2}\right)$ and $\left(A_{4}\right)$ holds. By [14, Theorem 3.1], $K$ is continuous and strictly monotone, hence the conditions $\left(A_{1}\right)$ and $\left(A_{3}\right)$ holds.

Moreover,

$$
\langle K(u), u\rangle=\int_{\Omega}|\nabla u(x)|^{p(x)} d x \geq 0,
$$

and equality holds only if $\nabla u(x)=0$ a.e., which implies that $u=0$ a.e.

\subsection{Existence results.}

Theorem 4.3. Assume that the conditions $\left(A_{1}\right)-\left(A_{4}\right)$ hold. Define the mapping $A$ as in (4.1). Let $f \in W^{-1, p^{\prime}(.)}(\Omega)$. If

$$
\liminf _{\|u\|_{1 . p(.)} \rightarrow \infty}\langle A(u)-f, u-\bar{u}\rangle \geq 0 \text { for some } \bar{u} \in W_{0}^{1, p(.)}(\Omega),
$$

then the problem (1.1) is almost solvable, i.e., $f \in \overline{A\left(W_{0}^{1, p(.)}(\Omega)\right)}$.

If

$$
\liminf _{\|u\|_{1, p(.)} \rightarrow \infty}\langle A(u)-f, u-\bar{u}\rangle>0 \text { for some } \bar{u} \in W_{0}^{1, p(.)}(\Omega),
$$

then the problem (1.1) is solvable, i.e., $f \in A\left(W_{0}^{1, p(.)}(\Omega)\right)$.

Proof. Suppose first that (4.15) holds. By theorem 4.1, $A \in \mathcal{F}^{a}$. If

$$
\inf _{\|u-\bar{u}\|_{1, p(.)}=R}\left(\langle A(u)-f, u-\bar{u}\rangle+\|A(u)-f\| \frac{R}{4 N \sqrt{N}}\right) \leq 0 \text { for all } R>0,
$$

then, by (4.15), there exists a sequence $\left(u_{n}\right) \subset W_{0}^{1, p(\cdot)}(\Omega)$ such that $\left\|u_{n}\right\|_{1, p(\cdot)} \rightarrow \infty$ and $\left\|A\left(u_{n}\right)-f\right\| \rightarrow 0$. Hence

Suppose that

$$
f \in \overline{A\left(W_{0}^{1, p(.)}(\Omega)\right)} .
$$

$$
\inf _{\|u-\bar{u}\|_{1, p(.)}=R}\left(\langle A(u)-f, u-\bar{u}\rangle+\|A(u)-f\| \frac{R}{4 N \sqrt{N}}\right)>0 \text { for some } R>0 .
$$

There exists a normalising map $K$ satisfying the assumptions of Corollary 4.1 with $b=\frac{R}{2 \sqrt{N}}$ and $a=\frac{R}{4 N \sqrt{N}}$.

Denote

$$
B_{R}(\bar{u})=\left\{u \in W_{0}^{1, p(\cdot)}(\Omega) /\|u-\bar{u}\|_{1, p(\cdot)} \leq R\right\} .
$$

By Corollary 4.1, $d\left(A, B_{R}(\bar{u}), f\right)=1$. By property (1) of the degree function, $f \in A\left(B_{R}(\bar{u})\right) \subset A\left(W_{0}^{1, p(.)}(\Omega)\right)$.

If (4.16) holds, then we clearly have

$$
\inf _{\|u-\bar{u}\|_{1, p(.)}=R}\left(\langle A(u)-f, u-\bar{u}\rangle+\|A(u)-f\| \frac{R}{4 N \sqrt{N}}\right)>0 \text { for some } R>0,
$$


and proceeding as above we obtain

$$
f \in A\left(W_{0}^{1, p(.)}(\Omega)\right) .
$$

\section{Acknowledgments}

We would like to thank the referees for their comments and suggestions on the manuscript.

\section{References}

[1] M. Ait Hammou and E. Azroul, Construction of a topological degree theory in Generalized Sobolev Spaces, J. of Univ. math., 1 no. 2 (2018), 116-129.

[2] M. Ait Hammou, E. Azroul and B. Lahmi, Existence of solutions for $p(x)$-Laplacian Dirichlet problem by Topological degree, Bull. Transilv. Univ. Braşov Ser. III, 11(60) no. 2 (2018), 29-38.

[3] M. Ait Hammou, E. Azroul and B. Lahmi, Topological degree methods for a Strongly nonlinear $p(x)$-elliptic problem, Rev. Colombiana Mat., 53 no. 1 (2019), 27-39.

[4] M. Ait Hammou and E. Azroul, Nonlinear Elliptic Problems in Weighted Variable Exponent Sobolev Spaces by Topological Degree, Proyecciones, 38 no. 4 (2019), 733-751.

[5] M. Ait Hammou and E. Azroul, Nonlinear elliptic boundary value problems by Topological degree, Recent Advances in Modeling, Analysis and Systems Control: Theoretical Aspects and Applications, Studies in Systems, Decision and Control 243, Springer Nature Switzerland AG 2020, doi.org /10.1007/978-3-030-261498_1, 1-13.

[6] M.L. Ahmed Oubeid, A. Benkirane, M. Sidi El Vally, Nonlinear elliptic equations involving measure data in Museilak-Orlicz-Sobolev spaces, J. of Abstract Diff. Equ. and App., 4 no. 1 (2013), 43-57.

[7] E. Azroul, H. Redwane and C. Yazough, Strongly nonlinear non homogeneous elliptic unilateral problems with $L^{1}$ data and no sign conditions, Electron. J. Differential Equations, 2012 no. 79 (2012), 1-20.

[8] S. Antontsev and S. Shmarev, Anisotropic parabolic equations with variable nonlinearity, Publ. Mat., 53 no. 2 (2009), 355-399.

[9] J. Berkovits, Extension of the Leray-Schauder degree for abstract Hammerstein type mappings, J. Differ. Equ., 234 (2007), 289-310.

[10] L.Boccardo and T.Gallouet, Nonlinear elliptic equations involving measure as data, J. Funct. Anal., 87 (1989), 149-169.

[11] L.E.J. Brouwer, Uber Abbildung von Mannigfaltigkeiten, Math. Ann., 71 (1912), 97-115.

[12] F.E. Browder, Fixed point theory and nonlinear problems., Bull. Am. Math. Soc., 9 (1983), 1-39.

[13] F.E. Browder, Degree of mapping for nonlinear mappings of monotone type., Proc. Nat. Acad. Sci. USA, 80 (1983), 1771-1773.

[14] K.C. Chang, Critical point theory and applications, Shanghai Scientific and Technology Press, Shanghai, 1986 (english).

[15] L. Dingien, P. Harjulehto, P. Hästö and M. Ruzicka, Lebesgue and Sobolev Spaces with Variable Exponents, Springer (2011).

[16] G.Dong, Elliptic equations with measure data in Orlicz spaces, Elec. J. of Diff. Equ., 2008 no. 76 (2008), 1-10.

[17] X. L. Fan and D. Zhao, On the Spaces $L^{p(x)}(\Omega)$ and $W^{m, p(x)}(\Omega)$; J. Math. Anal. Appl., 263 (2001), 424-446.

[18] O. Kováčik and J. Rákosník, On spaces $L^{p(x)}$ and $W^{1, p(x)}$, Czechoslovak Math. J., 41 (1991), 592-618.

[19] B. Lahmi, E. Azroul and K. El Haiti, Nonlinear degenerated elliptic problems with dual data and nonstandard growth, Math. reports, 20(70)no. 1 (2018), 81-91.

[20] J. Leray and J. Schauder, Topologie et equationes fonctionnelles, Ann. Sci. Ec. Norm. Super., 51 (1934), 45-78.

[21] R. Landes and V. Mustonen, Pseudo-monotne mappings in Orlicz-Sobolev spaces and nonlinear boundary value problems on unbounded domains, J. Math. Anal., 88 (1982), 25-36. 
[22] M. Ružička, Electrorheological fluids: modeling and mathematical theory, Lecture Notes in Mathematics 1748, Springer-verlag, Berlin (2000). 